\title{
Research Article \\ Role of Nampt and Visceral Adiposity in Esophagogastric Junction Adenocarcinoma
}

\author{
Haijun Li, ${ }^{1}$ E. Bai, ${ }^{2}$ Yong Zhang, ${ }^{1}$ Zhuoqi Jia, $^{1}$ Shicai He, ${ }^{3}$ and Junke Fu ${ }^{1}$ \\ ${ }^{1}$ Department of Thoracic Surgery, The First Affiliated Hospital of Xian Jiaotong University, Xian 710061, China \\ ${ }^{2}$ Department of Gynecology and Obstetrics, The First Affiliated Hospital of Xi'an Jiaotong University, Xian 710061, China \\ ${ }^{3}$ Department of General Surgery, The First Affiliated Hospital of Xi'an Jiaotong University, Xi'an 710061, China
}

Correspondence should be addressed to Junke Fu; fujunk-6612@126.com

Received 1 August 2016; Accepted 22 November 2016; Published 11 January 2017

Academic Editor: Nejat K. Egilmez

Copyright (C) 2017 Haijun Li et al. This is an open access article distributed under the Creative Commons Attribution License, which permits unrestricted use, distribution, and reproduction in any medium, provided the original work is properly cited.

\begin{abstract}
Nampt including eNampt and iNampt may contribute to mediating obesity-associated cancers. This study investigated the role of Nampt in esophagogastric junction adenocarcinoma (EGA), a cancer strongly correlated with obesity. Visceral adiposity was defined by waist circumference or VFA. eNampt in sera were measured by enzyme-linked immunosorbent assay. iNampt expression in EGA was determined by PCR, western blot, and immunohistochemistry. Sera eNampt were significantly elevated in these overweight and obese patients, especially for viscerally obese patients, and positively correlated with BMI, waist circumference, VFA, and also primary tumor, regional lymph nodes, and TNM stage $(P<0.05)$. iNampt expression in both the mRNA and protein levels was upregulated in EGAs $(P<0.05)$. iNampt staining was found primarily in the cytoplasm and nuclei and significantly associated with tumor, lymph nodes, and TNM stage and also correlated positively with serum eNampt, BMI, total fat area, VFA, superficial fat area, and waist circumference $(P<0.05)$. iNampt, eNampt, tumor, lymph nodes, and TNM stage correlated to the survival of EGAs, and iNampt expression and TNM stage affected the prognosis independently $(P<0.05)$. This study highlighted the association of eNampt/iNampt with visceral obesity and a potential impact on the biology of EGA.
\end{abstract}

\section{Introduction}

Esophagogastric junction adenocarcinoma (EGA) is commonly regarded as a separated tumor entirety of upper digestive tract malignant tumors [1]. The incidence of EGA has been dramatically increasing in both western and eastern countries and may be associated with the elevated ratio of overweight and obese populations [2]. Tumor en bloc removal combined with perigastric-mediastinal lymphadenectomy remains a mainstream treatment for the resectable EGAs [3]. Siewert types are the well-accepted classification of EGAs, simply as the distal esophagus (type I), true carcinoma of the cardia (type II), and subcardial carcinoma (type III) [4].

Nicotinamide phosphoribosyl transferase (Nampt) includes intracellular and extracellular Nampt (iNampt and eNampt, resp.). iNampt, a pleiotropic protein, acts as the rate-limiting enzyme in the salvage pathway for NAD biosynthesis and indirectly affects both cellular energetics and NAD-dependent enzymes [5]. iNampt is involved in different immune and metabolic disorders, especially cancer. iNampt was significantly upregulated or overexpressed in the genesis of a variety of malignant tumors, such as colorectal cancer [6], breast cancer [7], and gastric cancer [8].

Obesity, a growing health problem worldwide [9], correlates to an increased risk of numerous malignancies including colon cancer and breast cancer [5]. A meta-analysis has demonstrated that the overweight and obesity are strongly related to the risk of EGA [10], and some adipokines may prompt cancer cell survival and solid tumor progression [11]. The new adipokine, eNampt, mainly secreted by visceral fat, facilitated early B cell proliferation as pre-B-cell colonyenhancing factor (PBEF) at first [12] and drew considerable interest; after that it was described as an insulin-mimetic cytokine and termed as visfatin [13]. Although all three names (Nampt, PBEF, and visfatin) have been used in publications, Nampt has been approved as the official nomenclature of the protein and the gene by both the HUGO Gene Nomenclature Committee and the Mouse Genomic Nomenclature Committee. eNampt in serum is elevated with the increase 
of obesity, with many roles in physiology and pathology, and may well prove to be a significant mechanistic link in the network of cytokines influencing obesity-associated tumor progression. eNampt triggers numerous intracellular signalling pathways with varying temporal dynamics and can also stimulate multiple biological effects in a variety of cell types, especially tumor cells [14].

eNampt, as an cytokine, also functions as a NAD biosynthetic enzyme similar with iNampt. In the NAD biosynthetic pathway from nicotinamide, iNampt catalyzes the transfer of a phosphoribosyl group from 5-phosphoribosyl1-pyrophosphate to nicotinamide, forming nicotinamide mononucleotide (NMN) and pyrophosphate [15]. NMN is then converted to NAD with the help of nicotinamide mononucleotide adenylyltransferase (NMNAT) [16]. As such, these effects of eNampt can be attributed either to (i) its extracellular enzymic activity or to (ii) the binding and activation of a cell surface receptor [14]. Hence, eNampt/iNampt is so important and drew more and more interest, especially in the field of cancer. However, until now, data regarding the role of eNampt/iNampt and the association between eNampt and hematologic profile in patients with EGA is relatively limited. To investigate the role of eNampt and iNampt in EGA, we measured pretreatment plasma eNampt level as well as pretreatment hematologic profile and iNampt expression in the tumors in a Chinese population with EGA.

\section{Materials and Methods}

2.1. Patients and Tissues/Sera. Tumor samples and their paired adjacent nontumor tissues were collected, and the preoperative sera of 116 EGA patients were obtained from the First Affiliated Hospital of Xian Jiaotong University from June 2008 to May 2011. These EGA patients included 70 males and 46 females, and the median age of them was 61 years (ranging from 31 to 73 years). The study was approved by the Institutional Review Board of Xi'an Jiaotong University, and patients signed informed consent forms. All patients have not received preoperative chemotherapy or radiotherapy and have been without obvious weight loss $(<5 \%)$. The patients were grouped according to gender, age, Siewert types, differentiation, tumor, lymph nodes, and TNM stage. These EGA patients were followed up over a fiveyear period. Disease-specific survival of EGA patients was calculated as the time from diagnosis to the date of death filtering out the effect of mortality from other causes.

2.2. Anthropometry. Anthropomorphic data were measured before surgery by a single observer. Waist circumference was measured to the nearest $0.5 \mathrm{~cm}$ at the midpoint between the lower border of the rib cage and the iliac crest following gentle expiration. Central obesity was defined as a waist circumference greater than $80 \mathrm{~cm}$ in women and $94 \mathrm{~cm}$ in men [17]. Weight was measured to the nearest $0.1 \mathrm{~kg}$ with the patient dressed but without shoes or heavy outerwear. Height was measured to the nearest $0.5 \mathrm{~cm}$ with the patient barefoot. BMI was calculated as weight/height ${ }^{2}$. BMI was defined utilizing the World Health Organization definitions, with a BMI of
$20-24.9 \mathrm{~kg} / \mathrm{m}^{2}$ (normal), $25-29.9 \mathrm{~kg} / \mathrm{m}^{2}$ (overweight), and $>30 \mathrm{~kg} / \mathrm{m}^{2}$ (obese). All patients were asked about their body weight 12 months before diagnosis to allow an estimation of weight loss at diagnosis. Visceral fat area (VFA) and superficial fat area were calculated by computed tomography scanning of cross-sectional transverse images at the level of the third and fourth intervertebral discs [18]. The definition of visceral obesity was VFA exceeding $130 \mathrm{~cm}^{2}$ [19].

2.3. Enzyme-Linked Immunosorbent Assay. Serum eNampt level was measured utilizing commercially available eNampt ELISA kits (Linco Research, Inc., St. Charles, MO, USA) following the manufacturer's instructions in 96-well flexible microtiter plates. The wells were washed and biotinylated anti-eNampt antibody was added. After washing away unbound biotinylated antibody, HRP-conjugated streptavidin was pipetted to the wells. These wells were washed again, and a TMB substrate solution was used as the detecting agent. The OD of each well was read at $450 \mathrm{~nm}$.

2.4. RNA Expression Study. Total RNA was isolated from tissue utilizing the TRIzol reagent (Invitrogen, San Diego, CA) and then reverse-transcribed to cDNA with a cDNA synthesis kit (Takara Biochemicals, Japan) according to the manufacturer's protocols. Expression of Nampt mRNA was quantified by RT-PCR, and transcript levels were normalized to $\beta$-actin. Briefly, the reaction was run using an Icycler (Bio-Rad, Hercules, CA) with a preheating step at $95^{\circ} \mathrm{C}$ for $10 \mathrm{~min}$, followed by 30 cycles at $94.0^{\circ} \mathrm{C}$ for $30 \mathrm{sec}, 55.0^{\circ} \mathrm{C}$ for $30 \mathrm{sec}$, and $72^{\circ} \mathrm{C}$ for $1 \mathrm{~min}$. The primers used in the reaction were as follows: $\beta$-actin, forward: $5^{\prime}$-ATCGTGCGTGACATTAAGGAGAAG-3', reverse: $5^{\prime}$-AGGAAGGAAGGCTGGAAGAGTG-3'; Nampt, forward: $5^{\prime}$-AAGAGACTGCTGGCATAGGA-3', reverse: $5^{\prime}$-ACCACAGATACAGGCACTGA-3'. All cDNA samples were synthesized in parallel, and PCR reactions (the product, $\beta$-actin $179 \mathrm{bp}$; Nampt 181 bp) were run in triplicate.

2.5. Protein Extraction and Western Blotting. Protein from tissue was extracted with RIPA buffer, and the concentration was quantified via the BCA method (Pierce). An equal amount of protein was separated by SDS-PAGE and then transferred onto PDVF membranes (Millipore). Western blot analyses were then performed using anti-iNampt antibody (Santa Cruz Biotechnology, Santa Cruz, CA) or an anti- $\beta$ actin antibody (Santa Cruz Biotechnology). The blots were developed with chemiluminescence substrate solution from Pierce and exposed to X-ray film.

2.6. Immunohistochemistry (IHC). IHC was carried out on paraformaldehyde-fixed paraffin sections. Primary antiiNampt antibody (Santa Cruz Biotechnology) was used in the IHC with streptavidin peroxidase (SP) conjugated method. IHC was performed and the staining results for iNampt were semiquantitatively analyzed as previously reported [20], and sections with a total score of $>4$ were defined as exhibiting positive staining for iNampt. All histological analyses were carried out by three independent observers. 
2.7. Statistical Analysis. Values were presented as the mean $\pm \mathrm{SD}$. Continuous variables were compared using unpaired $t$ tests for normally distributed data and Mann-Whitney $U$ test otherwise. Association of categorical variables was assessed using $\chi^{2}$ test. Correlations between variables were assessed using the Spearman and Pearson correlation coefficients, as appropriate. Survival curves were estimated by the KaplanMeier method. Cox's proportional hazards regression analysis was done to estimate which factors might have a significant influence on survival. $P<0.05$ was considered statistically significant.

\section{Results}

3.1. eNampt Level in the Serum. Serum level of eNampt measured using ELISA correlated with obesity status and pathologic phase. Mean eNampt level was significantly higher in the serum of patients who were defined as obese by BMI $\left(7.81 \pm 1.54\right.$ versus $6.43 \pm 1.96 \mathrm{ng} / \mathrm{ml}$ for BMI $25 \mathrm{~kg} / \mathrm{m}^{2}$ or greater versus less than $25 \mathrm{~kg} / \mathrm{m}^{2}, P<0.001$ ), waist circumference $(7.82 \pm 1.36$ versus $6.50 \pm 2.02 \mathrm{ng} / \mathrm{ml}, P<$ $0.001)$, and VFA $(8.00 \pm 1.45$ versus $5.84 \pm 1.75 \mathrm{ng} / \mathrm{ml}, P<$ 0.001). eNampt level correlated with BMI $\left(r_{s}=0.34, P<\right.$ $0.001)$, waist circumference $\left(r_{s}=0.50, P<0.001\right)$, and VFA $\left(r_{s}=0.54, P<0.001\right)$; therefore VFA was the most close factor associated with eNampt.

Circulating eNampt level also correlated with primary tumor $\left(r_{s}=0.37, P<0.001\right)$, regional lymph nodes $\left(r_{s}=0.33\right.$, $P<0.001)$, and TNM stage $\left(r_{s}=0.42, P<0.001\right)$ but not with other clinicopathological factors including gender, age, Siewert types, and tumor differentiation $(P>0.05)$. Median disease-specific survival was significantly different between patients with the level of eNampt below and above the median $(36.00 \pm 0.69$ versus $32.00 \pm 1.04$ months, $P=0.002)$.

\subsection{Expression of iNampt in EGA Tissues and Clinicopatholog-} ical Characteristics. Expression of iNampt in EGAs and their adjacent nontumor tissues was analyzed in the mRNA and protein levels. Compared to their adjacent nontumor specimens, EGAs tissues had higher iNampt expression in both the mRNA and protein levels regardless of the background of obesity (Figure 1).

iNampt protein expression was also assessed semiqualitatively by immunohistochemical analysis. Immunoreactivity for iNampt was found primarily in the cytoplasm and nuclei (Figure 2). Expression of iNampt was significantly associated with tumor, lymph nodes, and TNM stage (Table $1, P<0.05$ ). However, there were no correlations to gender, age, Siewert types, and tumor differentiation $(P>0.05)$. iNampt protein expression in these tumors was positively correlated with sera eNampt of these patients $(r=0.80, P<0.001)$. There was a higher proportion of patients with lymphatic invasion in those with greater than median iNampt expression (79.69 versus $25.00 \%, P<0.001)$.

3.3. iNampt Protein Expression in the Tumors and Their Obesity Status. iNampt expression in those tumor specimens from EGAs was also evaluated in consideration of metabolic features. iNampt expression correlated moderately with all markers of obesity (Table 2). Patients in the highest iNampt expression quartile had significantly higher BMI $(P=0.018)$, wider total fat area $(P<0.001)$, larger VFA $(P<0.001)$, bigger superficial fat area $(P=0.004)$, and upper waist circumference $(P<0.001)$ than those in the lowest quartile (Table 3). Viscerally obese patients were 4.1 times more likely to be within the highest quartile of iNampt expression than the lowest $\left(\chi^{2}=19.66, P<0.001\right)$. Patients classified as viscerally obese had significantly higher tumor iNampt expression than patients who were not viscerally obese (7.31 \pm 3.04 versus $4.34 \pm 2.64, P<0.001)$.

3.4. Nampt, Obesity Status, Clinicopathological Features, and Survival. Disease-specific survival was significantly reduced in those with positive compared with negative iNampt staining $(33.59 \pm 1.21$ versus $41.85 \pm 1.76$ months, $P<$ 0.001 , Figure 3). On univariable regression analysis, iNampt (positive versus negative expression), eNampt (below versus above the median), tumor (T1 and T2 versus T3 and T4), lymph nodes (N0 versus N1 and N2), and TNM stage (I and II versus III and IV) correlated to the survival of EGAs, but BMI, waist circumference, and VFA were not related to the prognosis of these patients with EGA. Positive tumor iNampt staining was associated with death from EGA compared with iNampt-negative tumors $(\mathrm{HR}=6.78, P<0.001)$, and TNM stages I and II were associated with death from EGA compared with stages III and IV $(\mathrm{HR}=0.06, P<$ 0.001). On multivariable analysis, both iNampt expression and TNM stage were independently associated with the survival (Table 4 ).

\section{Discussion}

A variety of clinical studies have linked adiposity with increased cancer incidence, progression, and metastasis, and adipose tissue is now being credited with both systemic and local effects on tumor development and survival [21]. Elucidating the mechanisms linking obesity and EGA is so complex by the numerous influences of obesity. Obesity primarily manifests the accumulation of adipose tissue. Adipose tissue was regarded as an insulating and mechanically supportive site of energy storage for a long time and now draws more and more attention for secreting a variety of adipokines [22]. Adipokines are polypeptide growth factors and cytokines and are secreted mainly by white adipose tissue preadipocytes and mature adipocytes. These potent adipokines such as resistin, leptin, and adiponectin are involved in cell growth, proliferation, cell cycle control, and angiogenesis [23] and could play a significant role in facilitating tumor growth and metastasis.

The new adipokine, eNampt, is not only produced by adipocytes and pre-B-cells but also readily detectable in conditioned media from cultures of most cell types, including cancer cells, such as hepatoma cells, colorectal cancer cells, breast cancer cells, melanoma cells, prostate cancer cells, and cervical cancer cells [14]. eNampt acts as a cytokine and demonstrates its biological potential as a putative paracrine 


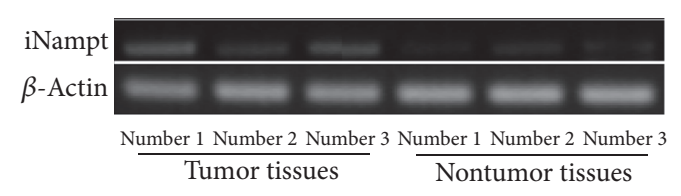

(a)

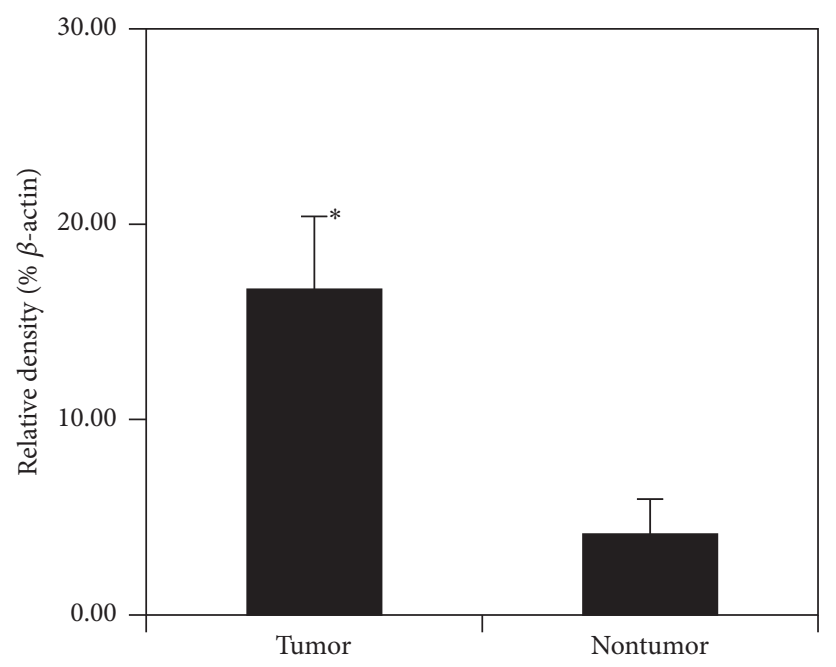

(b)

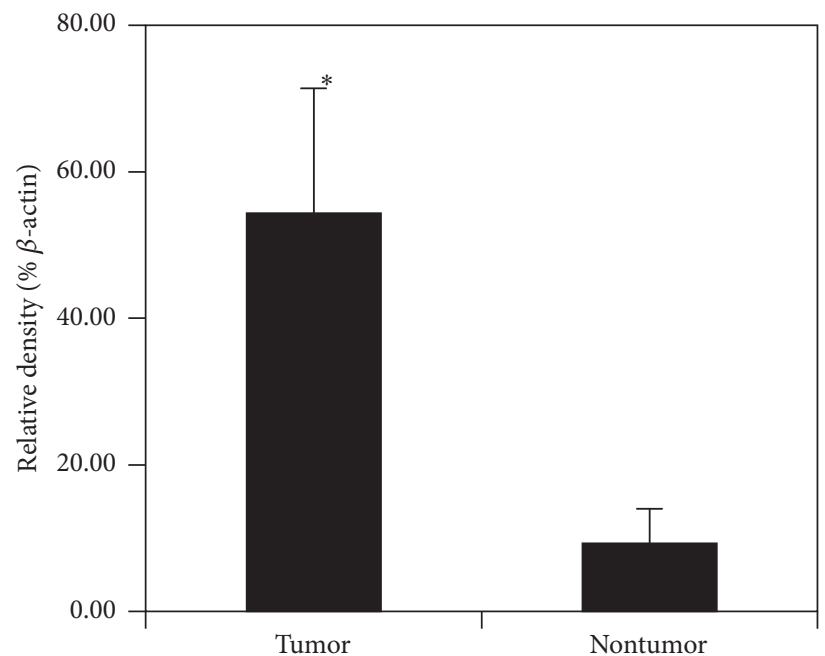

(d)

FIGURE 1: iNampt expression in EGAs tissues and their adjacent nontumor tissues ( $n=5 ; 3$ of 5 representative images were shown). Compared to these adjacent nontumor specimens, EGA had upregulated iNampt expression in the mRNA level ((a) and (b), $16.66 \pm 3.74 \%$ versus $4.14 \pm$ $\left.1.79 \%,{ }^{*} P=0.001<0.05\right)$ and protein level $\left((\mathrm{c})\right.$ and $(\mathrm{d}), 54.34 \pm 17.05 \%$ versus $\left.9.32 \pm 4.69 \%,{ }^{*} P=0.003<0.05\right)$.

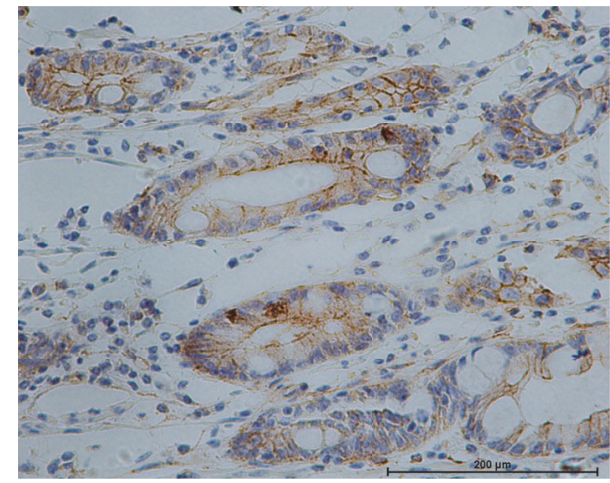

(a)

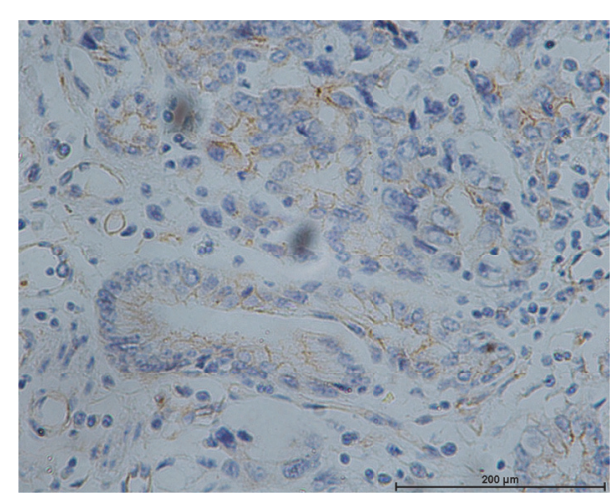

(b)

FIGURE 2: The protein expression of iNampt in these EGA specimens was analyzed by immunohistochemical staining $(\times 400)$. iNampt expression was significantly upregulated in these tumors from obese patients, especially visceral obesity (a), compared to those nonobese patients (b) $(7.31 \pm 3.04$ versus $4.34 \pm 2.64, P<0.001)$. 
TABLE 1: Clinical correlation of iNampt protein expression in EGA.

\begin{tabular}{|c|c|c|c|c|c|}
\hline \multirow{2}{*}{$\begin{array}{l}\text { Clinicopathological } \\
\text { features }\end{array}$} & \multirow{2}{*}{ Categories } & \multicolumn{2}{|c|}{ iNampt expression } & \multirow{2}{*}{$\chi^{2}$} & \multirow{2}{*}{$P$} \\
\hline & & - & + & & \\
\hline \multirow{2}{*}{ Gender } & Male & 31 & 39 & \multirow{2}{*}{0.21} & \multirow{2}{*}{0.89} \\
\hline & Female & 21 & 25 & & \\
\hline \multirow[b]{2}{*}{ Age (years) } & $<60$ & 27 & 23 & \multirow{2}{*}{2.99} & \multirow[b]{2}{*}{0.08} \\
\hline & $\geq 60$ & 25 & 41 & & \\
\hline \multirow{3}{*}{ Siewert types } & I & 8 & 19 & \multirow{3}{*}{3.41} & \multirow{3}{*}{0.18} \\
\hline & II & 27 & 26 & & \\
\hline & III & 17 & 19 & & \\
\hline \multirow{2}{*}{ Differentiation } & \multirow{2}{*}{$\mathrm{G} 1$ \& G2G3 \& G4 } & 25 & 33 & \multirow{2}{*}{0.14} & \multirow{2}{*}{0.71} \\
\hline & & 27 & 31 & & \\
\hline \multirow{2}{*}{ Tumor } & \multirow{2}{*}{$\mathrm{T} 1 \& \mathrm{~T} 2 \mathrm{~T} 3$ \& $\mathrm{T} 4$} & 38 & 25 & \multirow{2}{*}{13.38} & \multirow{2}{*}{$<0.001^{*}$} \\
\hline & & 14 & 39 & & \\
\hline Lymph nodes & $\begin{array}{c}\mathrm{N} 0 \\
\mathrm{~N} 1 \& \mathrm{~N} 2\end{array}$ & 39 & 13 & \multirow{2}{*}{34.69} & \multirow{2}{*}{$<0.001^{*}$} \\
\hline \multirow{3}{*}{ TNM stage } & N1 \& N2 & $\begin{array}{l}13 \\
18\end{array}$ & 51 & & \\
\hline & II & 26 & $\begin{array}{c}4 \\
16\end{array}$ & \multirow[t]{2}{*}{35.35} & \multirow[t]{2}{*}{$<0.001^{*}$} \\
\hline & III & 8 & 44 & & \\
\hline
\end{tabular}

TNM: tumor node metastasis.

* Statistically significant.

TABLE 2: Correlations between relative quantification values for iNampt and measures of obesity.

\begin{tabular}{lcc}
\hline Measures of obesity & \multicolumn{2}{c}{ iNampt expression } \\
\hline Body mass index & $r_{s}$ & $P$ \\
Waist circumference & 0.25 & $0.007^{*}$ \\
Visceral fat area & 0.40 & $<0.001^{*}$ \\
Superficial fat area & 0.49 & $<0.001^{*}$ \\
Total fat area & 0.33 & $<0.001^{*}$ \\
\hline
\end{tabular}

${ }^{*}$ Statistically significant.

and autocrine factor. eNampt may promote tumor progression in a large number of cancers including breast cancer $[24,25]$ and prostate cancer [26]. Furthermore, eNampt levels in sera were associated with tumor progression in malignant astrocytomas [27], gastric cancer [28], and colorectal cancer [29]. This study demonstrated the close relationship between EGA and eNampt and showed that eNampt was significantly associated with tumor, regional lymph nodes, and TNM stage, indicating that EGA cells released eNampt into the sera. This study also revealed that eNampt was significantly correlated to these markers of obesity including BMI, waist circumference, and VFA, indicating that adipose tissue secreted eNampt into the sera. These results were consistent with the above-mentioned notion. eNampt provided by these two sources and others might recognize some cell surface receptor and also acts as a NAD biosynthetic enzyme [14]. As such, eNampt may promote EGA development by increasing intracellular $\mathrm{NAD}^{+}$content, which directly affects the ability of Sirtl or by other ways.

In addition, iNampt is closely related to malignant tumors including gastric cancer [8] and was also overexpressed in

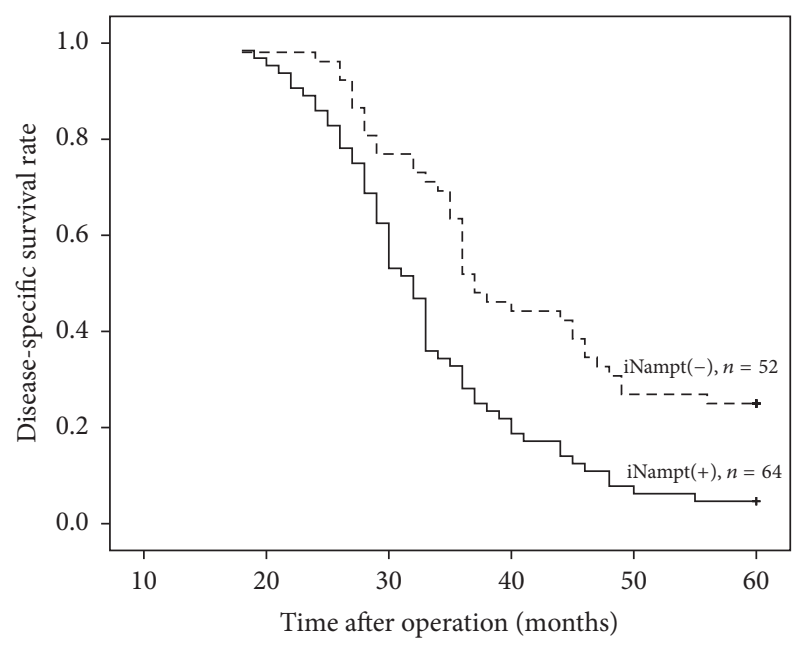

FIGURE 3: Kaplan-Meier analysis of disease-specific survival of patients with EGA relative to iNampt expression.

the EGA specimens in this study. This study also showed that expression of iNampt was significantly associated with tumor, regional lymph nodes, and TNM stage but not correlated with gender, age, Siewert types, and tumor differentiation. It was very interesting in this study that iNampt expression seems to be age-dependent. Further analysis showed that more patients who were older than 60 years were viscerally obese compared to others (39/66 versus $19 / 50, P<0.05)$, although these two groups had no difference in the BMI and waist, and the expression of iNampt in EGA tissues was not significantly correlated with age of patients $\left(r_{s}=0.17, P=\right.$ $0.166)$.

The novel findings of this study that serum eNampt were positively correlated with the expression of iNampt in 
TABLE 3: Differences in obesity status between tumors with lowest- versus highest-quartile iNampt expression.

\begin{tabular}{lccc}
\hline Obesity status & iNampt expression & & \\
& Lowest-quartile & Highest-quartile & \\
\hline Body mass index $\left(\mathrm{kg} / \mathrm{m}^{2}\right)$ & $24.00 \pm 1.76$ & $25.38 \pm 2.26$ & \\
Waist circumference $(\mathrm{cm})$ & $81.26 \pm 4.01$ & $87.87 \pm 4.45$ & $0.018^{*}$ \\
Total fat area $\left(\mathrm{cm}^{2}\right)$ & $184.70 \pm 22.16$ & $215.56 \pm 17.11$ & $<0.001^{*}$ \\
Visceral fat area $\left(\mathrm{cm}^{2}\right)$ & $118.50 \pm 14.62$ & $141.18 \pm 9.65$ & $<0.001^{*}$ \\
Superficial fat area $\left(\mathrm{cm}^{2}\right)$ & $66.20 \pm 9.74$ & $74.39 \pm 9.78$ & $<0.001^{*}$ \\
\hline
\end{tabular}

${ }^{*}$ Statistically significant.

TABLE 4: Cox regression analysis of factors associated with death from EGA.

\begin{tabular}{|c|c|c|c|c|}
\hline & \multicolumn{2}{|c|}{ Univariable analysis } & \multicolumn{2}{|c|}{ Multivariable analysis } \\
\hline & HR $(95 \% \mathrm{CI})$ & $P$ & HR (95\% CI) & $P$ \\
\hline iNampt (positive versus negative expression) & $6.78(1.81-25.33)$ & $0.002^{*}$ & $3.00(1.03-8.69)$ & $0.043^{*}$ \\
\hline eNampt (below versus above the median) & $0.15(0.04-0.55)$ & $0.002^{*}$ & $0.38(0.14-1.01)$ & 0.052 \\
\hline Tumor (T1 \& T2 versus T3 \& T4) & $0.14(0.03-0.64)$ & $0.004^{*}$ & $0.76(0.46-1.25)$ & 0.759 \\
\hline Lymph nodes (N0 versus N1 \& N2) & $0.09(0.02-0.41)$ & $<0.001^{*}$ & $0.63(0.34-1.19)$ & 0.156 \\
\hline TNM stage (I \& II versus III \& IV) & $0.06(0.01-0.46)$ & $<0.001^{*}$ & $0.20(0.09-0.43)$ & $<0.001^{*}$ \\
\hline
\end{tabular}

TNM: tumor node metastasis.

${ }^{*}$ Statistically significant.

the tumors were consistent with the notion that changed adipokines and other growth factors following obesity formation may promote cancer cell survival and solid tumor progression [30]. In this study, the altered iNampt expression was associated with changes in disease-specific survival. iNampt was correlated positively with serum eNampt, and eNampt were significantly elevated in the sera of overweight and obese patients (BMI $>25 \mathrm{~kg} / \mathrm{m}^{2}$, which was defined as obese in china), especially for viscerally obese patients, and positively correlated with BMI, waist circumference, and VFA. Hence, iNampt was associated with all markers of obesity including BMI, total fat area, VFA, superficial fat area, and waist circumference. The fact that median disease-specific survival was significantly longer in these patients below the median eNampt level than above that was consistent with the results that survival was shorter in those with positive iNampt staining than negative expression. This study supports the hypothesis that obesity status influences eNampt/iNampt expression, but it is noticeable that all markers of obesity were not correlated to the survival of these patients in the study.

For valid evidence, the patient cohort was very restricted to those undergoing surgical treatment with curative intent and without obvious weight loss in this study. iNampt, eNampt, tumor, regional lymph nodes, and TNM stage correlated to the survival of EGAs, and iNampt expression and TNM stage were independently associated with the survival. Hence, further studies are required to determine the downstream impact of this altered eNampt and iNampt expression on the pathways that impact survival, proliferation, and apoptosis.

\section{Conclusion}

iNampt expression was independent prognostic indicator for EGA patients. This study highlighted the association of
eNampt/iNampt with visceral obesity and a potential impact on the biology of EGA. Targeting eNampt/iNampt may have a rationale in future studies.

\section{Competing Interests}

The authors declare that they have no conflict of interests.

\section{References}

[1] H.-I. Kim, J.-H. Cheong, K. J. Song et al., "Staging of adenocarcinoma of the esophagogastric junction: comparison of AJCC 6th and 7th gastric and 7th esophageal staging systems," Annals of Surgical Oncology, vol. 20, no. 8, pp. 2713-2720, 2013.

[2] I. Oda, S. Abe, C. Kusano et al., "Correlation between endoscopic macroscopic type and invasion depth for early esophagogastric junction adenocarcinomas," Gastric Cancer, vol. 14, no. 1, pp. 22-27, 2011.

[3] T. Matsuda, H. Takeuchi, S. Tsuwano et al., "Optimal surgical management for esophagogastric junction carcinoma," General Thoracic and Cardiovascular Surgery, vol. 62, no. 9, pp. 560-566, 2014.

[4] J. R. Siewert and H. J. Stein, "Classification of adenocarcinoma of the oesophagogastric junction," British Journal of Surgery, vol. 85, no. 11, pp. 1457-1459, 1998.

[5] I. Vucenik and J. P. Stains, "Obesity and cancer risk: evidence, mechanisms, and recommendations," Annals of the New York Academy of Sciences, vol. 1271, pp. 37-43, 2012.

[6] J. R. Van Beijnum, P. T. M. Moerkerk, A. J. Gerbers et al., "Target validation for genomics using peptide-specific phage antibodies: a study of five gene products overexpressed in colorectal cancer," International Journal of Cancer, vol. 101, no. 2, pp. 118-127, 2002.

[7] M. A. A. K. Folgueira, D. M. Carraro, H. Brentani et al., "Gene expression profile associated with response to doxorubicinbased therapy in breast cancer," Clinical Cancer Research, vol. 11, no. 20, pp. 7434-7443, 2005. 
[8] T.-Q. Bi, X.-M. Che, X.-H. Liao et al., "Overexpression of Nampt in gastric cancer and chemopotentiating effects of the Nampt inhibitor FK866 in combination with fluorouracil," Oncology Reports, vol. 26, no. 5, pp. 1251-1257, 2011.

[9] K. M. Flegal, D. Kruszon-Moran, M. D. Carroll, C. D. Fryar, and C. L. Ogden, "Trends in obesity among adults in the United States, 2005 to 2014," The Journal of the American Medical Association, vol. 315, no. 21, pp. 2284-2291, 2016.

[10] F. Turati, I. Tramacere, C. La Vecchia, and E. Negri, "A metaanalysis of body mass index and esophageal and gastric cardia adenocarcinoma," Annals of Oncology, vol. 24, no. 3, pp. 609617, 2013.

[11] A. Gucalp, N. M. Iyengar, C. A. Hudis, and A. J. Dannenberg, "Targeting obesity-related adipose tissue dysfunction to prevent cancer development and progression," Seminars in Oncology, vol. 43, no. 1, pp. 154-160, 2016.

[12] B. Samal, Y. Sun, G. Stearns, C. Xie, S. Suggs, and I. Mcniece, "Cloning and characterization of the cDNA encoding a novel human pre-B-cell colony-enhancing factor," Molecular and Cellular Biology, vol. 14, no. 2, pp. 1431-1437, 1994.

[13] Q. Sun, L. Li, R. Li et al., "Overexpression of visfatin/PBEF/ Nampt alters whole-body insulin sensitivity and lipid profile in rats," Annals of Medicine, vol. 41, no. 4, pp. 311-320, 2009.

[14] A. A. Grolla, C. Travelli, A. A. Genazzani, and J. K. Sethi, "Extracellular nicotinamide phosphoribosyltransferase, a new cancer metabokine," British Journal of Pharmacology, vol. 173, no. 14, pp. 2182-2194, 2016.

[15] J. R. Revollo, A. A. Grimm, and S.-I. Imai, "The regulation of nicotinamide adenine dinucleotide biosynthesis by Nampt/PBEF/visfatin in mammals," Current Opinion in Gastroenterology, vol. 23, no. 2, pp. 164-170, 2007.

[16] A. Garten, S. Petzold, A. Körner, S.-I. Imai, and W. Kiess, "Nampt: linking NAD biology, metabolism and cancer," Trends in Endocrinology \& Metabolism, vol. 20, no. 3, pp. 130-138, 2009.

[17] K. G. M. M. Alberti, R. H. Eckel, S. M. Grundy et al., "Harmonizing the metabolic syndrome: a joint interim statement of the international diabetes federation task force on epidemiology and prevention; national heart, lung, and blood institute; american heart association; world heart federation; international atherosclerosis society; and international association for the study of obesity," Circulation, vol. 120, no. 16, pp. 1640-1645, 2009.

[18] P. Beddy, J. Howard, C. McMahon et al., "Association of visceral adiposity with oesophageal and junctional adenocarcinomas," British Journal of Surgery, vol. 97, no. 7, pp. 1028-1034, 2010.

[19] C. L. Donohoe, S. L. Doyle, S. McGarrigle et al., "Role of the insulin-like growth factor 1 axis and visceral adiposity in oesophageal adenocarcinoma," British Journal of Surgery, vol. 99, no. 3, pp. 387-396, 2012.

[20] H.-J. Li, X.-M. Che, W. Zhao, S.-C. He, Z.-L. Zhang, and R. Chen, "Diet-induced obesity potentiates the growth of gastric cancer in mice," Experimental and Therapeutic Medicine, vol. 4, no. 4, pp. 615-620, 2012.

[21] H. C. Gusky, J. Diedrich, O. A. Macdougald, and I. Podgorski, "Omentum and bone marrow: how adipocyte-rich organs create tumour microenvironments conducive for metastatic progression," Obesity Reviews, vol. 17, no. 11, pp. 1015-1029, 2016.

[22] K. M. Nieman, I. L. Romero, B. Van Houten, and E. Lengyel, "Adipose tissue and adipocytes support tumorigenesis and metastasis," Biochimica et Biophysica Acta - Molecular and Cell Biology of Lipids, vol. 1831, no. 10, pp. 1533-1541, 2013.
[23] T. Deng, C. J. Lyon, S. Bergin, M. A. Caligiuri, and W. A. Hsueh, "Obesity, inflammation, and cancer," Annual Review of Pathology: Mechanisms of Disease, vol. 11, pp. 421-449, 2016.

[24] S.-R. Kim, H.-J. Park, Y.-H. Bae et al., "Curcumin downregulates visfatin expression and inhibits breast cancer cell invasion," Endocrinology, vol. 153, no. 2, pp. 554-563, 2012.

[25] J. G. Kim, E. O. Kim, B. R. Jeong et al., "Visfatin stimulates proliferation of MCF-7 human breast cancer cells," Molecules and Cells, vol. 30, no. 4, pp. 341-345, 2010.

[26] S. T. Patel, T. Mistry, J. E. P. Brown et al., "A novel role for the adipokine visfatin/pre-B cell colony-enhancing factor 1 in prostate carcinogenesis," Peptides, vol. 31, no. 1, pp. 51-57, 2010.

[27] P. S. Reddy, S. Umesh, B. Thota et al., "PBEF1/NAmPRTase/ Visfatin: a potential malignant astrocytoma/glioblastoma serum marker with prognostic value," Cancer Biology and Therapy, vol. 7, no. 5, pp. 663-668, 2008.

[28] T. E. Nakajima, Y. Yamada, T. Hamano et al., "Adipocytokine levels in gastric cancer patients: resistin and visfatin as biomarkers of gastric cancer," Journal of Gastroenterology, vol. 44, no. 7, pp. 685-690, 2009.

[29] T. E. Nakajima, Y. Yamada, T. Hamano et al., "Adipocytokines as new promising markers of colorectal tumors: adiponectin for colorectal adenoma, and resistin and visfatin for colorectal cancer," Cancer Science, vol. 101, no. 5, pp. 1286-1291, 2010.

[30] S. Yakar, N. P. Nunez, P. Pennisi et al., "Increased tumor growth in mice with diet-induced obesity: impact of ovarian hormones," Endocrinology, vol. 147, no. 12, pp. 5826-5834, 2006. 


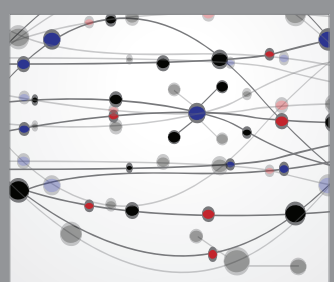

The Scientific World Journal
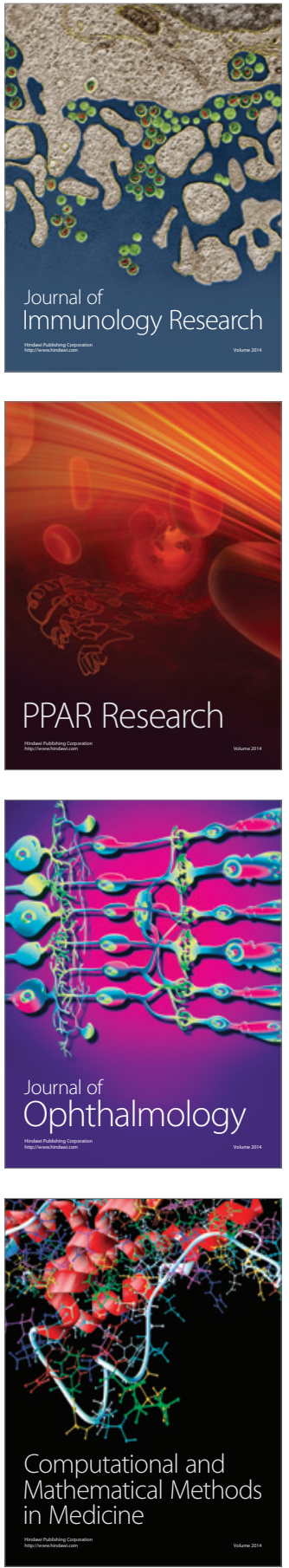

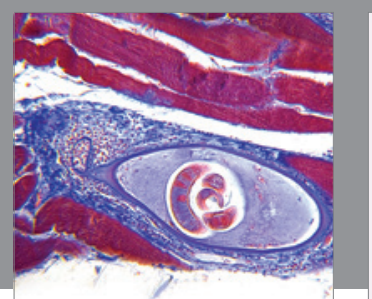

Gastroenterology Research and Practice
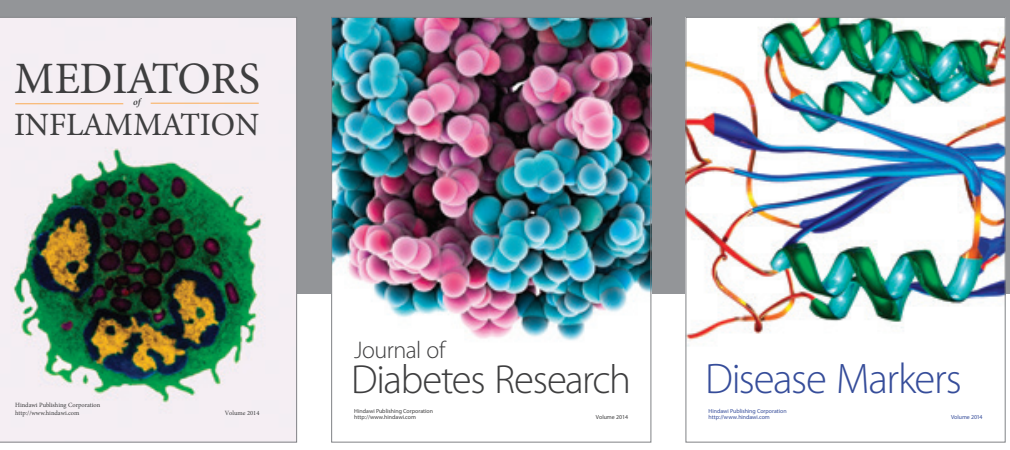

Disease Markers

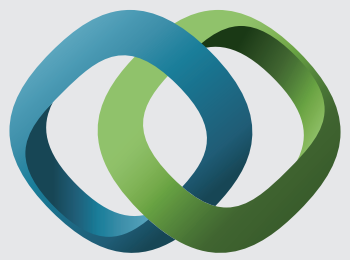

\section{Hindawi}

Submit your manuscripts at

https://www.hindawi.com
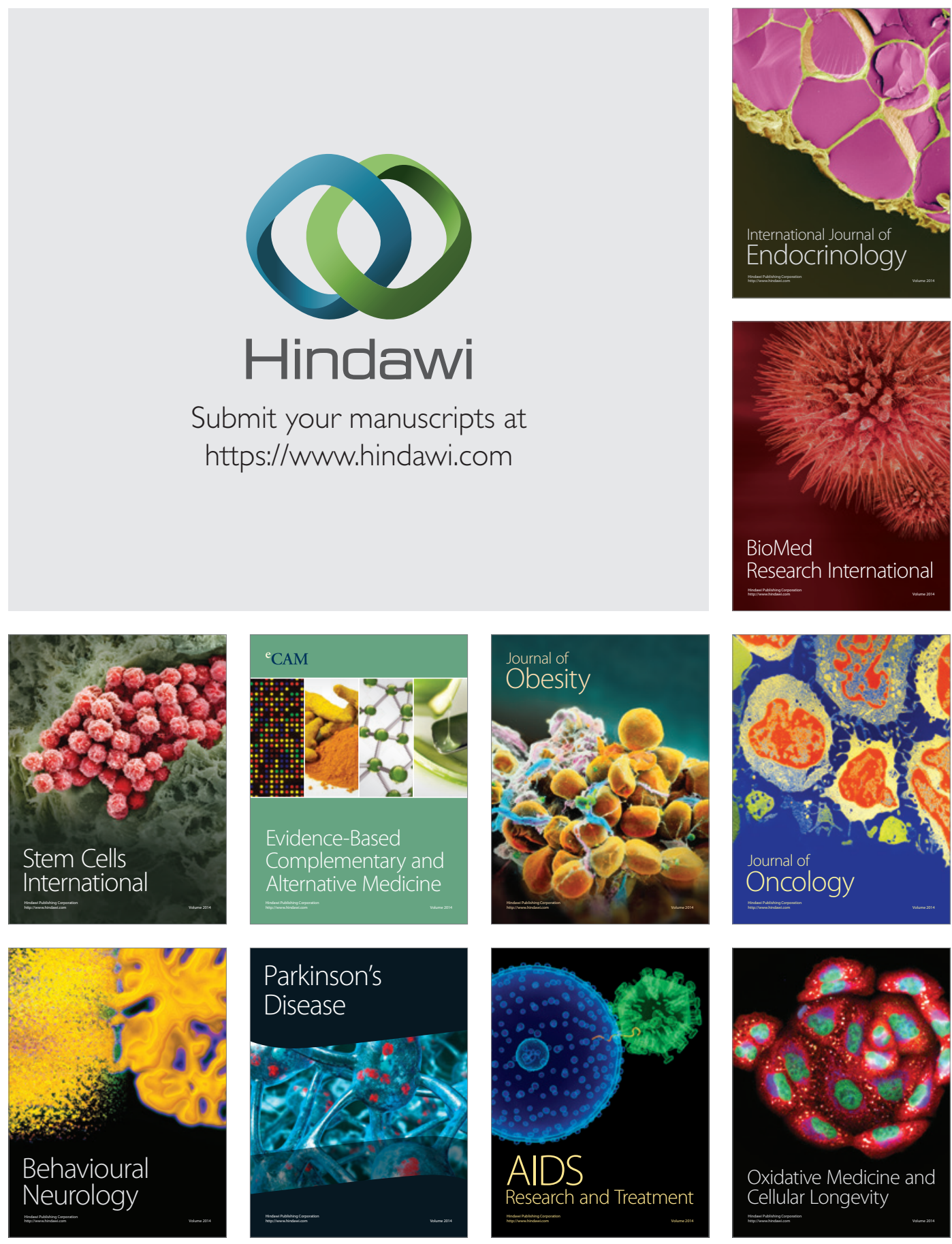